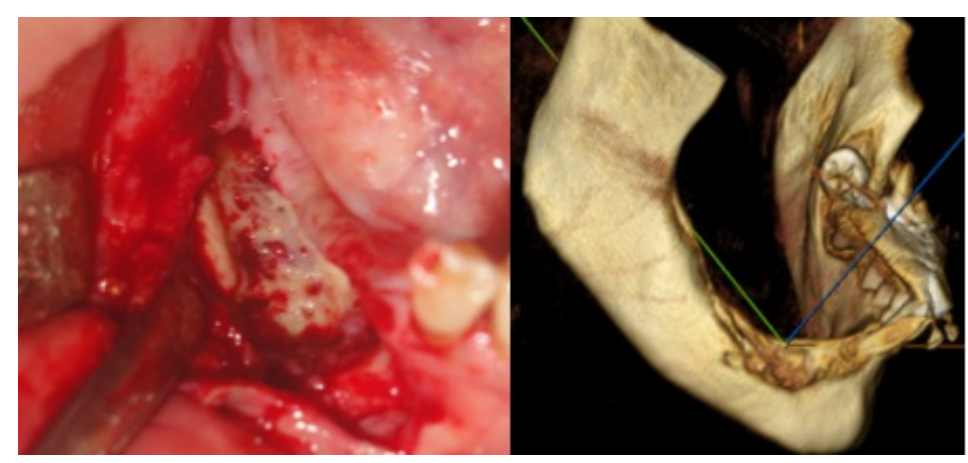

\title{
M-TOR INHIBITORS \& MRONJ. POSSIBLE ROLE OF EVEROLIMUS IN A CASE OF RECURRENT MANDIBULAR OSTEONECROSIS
}

\author{
FRANCESCO DELLA FERRERA ${ }^{1}$, Paolo Appendino ${ }^{1}$, balthazar fornaca, lorenzo basano \\ 1 Azienda Ospedaliera Ordine Mauriziano di Torino
}

Funding: The author(s) received no specific funding for this work.

Potential competing interests: The author(s) declared that no potential competing interests exist.

\section{Abstract}

Introduction: Concomitant therapy with antiresorptive/antiangiogenic drugs is common in cancer management and is reported to have a modifying effect on MRONJ development. M-TOR inhibitors have antiangiogenic and immunosuppressive properties.

Case report: A case of a 62 years old female with history of MRONJ who developed a recurrent mandibular osteonecrosis following few administration of sole Everolimus is reported here.

Introduction: MRONJ (Medication Related Osteonecrosis of the Jaws) is an adverse event in patient exposed to antiresorptive agents (Bisphosphonates-BP, Denosumab-Dmab) for prevention of skeletal-related events. Contemporary administration of antiresorptive/antiangiogenic drugs is common in cancer management. Several reports suggest a modifying effect of concomitant antiresorptive/antiangiogenic therapy on MRONJ outbreak ${ }^{1}$. Several molecules with antiangiogenic activity have been introduced in recent years. M-TOR inhibitors (eg. everolimus-Emus) have antiangiogenic and immunosuppressive properties ${ }^{1,2}$. A case with history of MRONJ in which the administration of Emus was soon followed by recurrence of the disease is presented.

Case report: A female 62 y.o. patient diagnosed of metastatic breast cancer (vertebrae) started Dmab therapy in 2015 with no previous employment of BP. In March 2017 the patient was referred to our department for pain and swelling in right mandibular body. Dmab was stopped and a MRONJ lesion was definitely diagnosed (SPECT-CT) in April. The lesion was 
probably determined by sore spot of removable denture worn by this partially edentulous patient. Firstly treated with conservative treatment, surgical intervention with significant resolution of symptoms was performed in June 2017. Wound dehiscence soon appeared in the mesial part of the surgical access but showed decreasing depth and re-epitelization during follow-up. Despite delayed wound healing no contraindications to restart oncologic treatments was then given. Healing conditions were still good in July 2018. The patient never restarted Dmab whereas in October 2018 Emus was prescribed for oncologic reasons. In January 2019 right mandibular swelling and pain with recurrence of wound dehiscence in the same area of previous MRONJ were observed. Emus was stopped and after conservative treatment, surgical intervention was performed in May 2019. After 1-year follow-up period the patient is free from signs/symptoms of MRONJ; a new prosthetic rehabilitation could be performed. Healing stability is confirmed by clinical and radiological means (CT scan).

Discussion: Association of MRONJ and antiangiogenics is well-known ${ }^{1}$. Osteonecrotic complications are also reported in patients solely treated with $\mathrm{m}$-TOR inhibitors ${ }^{2}$. This patient developed MRONJ recurrence after few administrations of Emus. MRONJ best treatment choice and healing criteria are still a matter of debate. Symptom regression, absence of bone exposure and mucosal coverage are generally considered clinical signs of healing eventually implemented by radiological findings. Drug-holidays despite their claimed usefulness in promoting healing after oral surgery procedures are always considered for every single case and shortened as much as possible given their implications in cancer management and its related events ${ }^{3}$. Considering previous healing stability of surgical site, time from first intervention $(>1$ year) and prolonged Dmab suspension (19 months) a possible precipitating effect of Emus therapy on MRONJ recurrence was supposed in this case. Considering growing list of medications possibly responsible for MRONJ, careful follow-up in cancer patient (specially with history of MRONJ) is crucial for prevention/interception of osteonecrotic complications.

\section{References:}

1. Pimolbutr K. et al. Osteonecrosis of the Jaw Associated with Antiangiogenics in Antiresorptive-Naive Patient: A Comprehensive Review of the Literature Biomed Res. Int. 18 Apr23;2018:8071579.

2. Eguia A,et al. Review and update on drugs related to the development of osteonecrosis of the jaw. Med Oral Patol Oral Cir Bucal. 2020 Jan 1;25 (1):e71-83.

3. Ramaglia L. et al. Stage-specific therapeutic strategies of medication-related osteonecrosis of the jaws: a systematic review and meta-analysis of the drug suspension protocol. Clin Oral Investig. 2018 Mar;22(2):597-615. 